\title{
Fabrication of Polymer Magnetic Nanocomposites Containing Carbon Nanoparticles Doped with Cobalt Nanoclusters and Study Their Conductivity, Self-Healing and Adhesion Properties
}

\author{
G. I. Mamniashvili', D. I. Gventsadze' ${ }^{2}$, L. N. Rukhadze' ${ }^{2}$, L. A. Maisuradze ${ }^{3}$ \\ ${ }^{1}$ Andronikashvili Institute of Physics, Ivane Javakhishvili Tbilisi State University, Tbilisi, Georgia \\ ${ }^{2}$ Georgian Technical University, Tbilisi, Georgia \\ ${ }^{3}$ Georgian Aviation University, Tbilisi, Georgia \\ Email:mgrigor@rocketmail.com
}

How to cite this paper: Mamniashvili, G.I., Gventsadze, D.I., Rukhadze, L.N. and Maisuradze, L.A. (2020) Fabrication of Polymer Magnetic Nanocomposites Containing Carbon Nanoparticles Doped with Cobalt Nanoclusters and Study Their Conductivity, Self-Healing and Adhesion Properties. World Journal of Condensed Matter Physics, 10, 118-134.

https://doi.org/10.4236/wjcmp.2020.103008

Received: July 2, 2020

Accepted: August 3, 2020

Published: August 6, 2020

Copyright () 2020 by author(s) and Scientific Research Publishing Inc. This work is licensed under the Creative Commons Attribution International License (CC BY 4.0).

http://creativecommons.org/licenses/by/4.0/ (c) (i) Open Access

\begin{abstract}
The technology of fabrication of polymer nanocomposites on basis of carbon nanoparticles doped with cobalt clusters, synthesized by original Chemical Vapore Deposition (CVD) technology developed by authors, was elaborated. Carbon shells provide both the protection of ferromagnetic impurities from aggressive environment and new unique properties to the hybride nanostructures. The self-assembling of magnetic clusters coated by carbon shells presents just such example which could be used in the contemporary materials, for example, in strong magnets, analytic instruments (nuclear magnetic resonance tomographs) and nanosensors. Their good conductivity, self-healing and adhesion properties were demonstrated by applying the combined action of temperature, pressure, steady and alternating magnetic fields to stimulate diffusion of magnetic nanoparticles in direction to defect sites. Due to these properties fabricated magnetic polymer nanocomposites could have perspective for potential.
\end{abstract}

\section{Keywords}

Magnetic Carbon Nanopowder, Polymer Composite, Stimulated Diffusion, Self-Healing, Self-Organization, Resistance

\section{Introduction}

In the last decade, the investigation of such new nanostructure forms of carbon 
as nanoparticles, nanotubes, nanothreads became very topical. This is related with the fact that the pointed nanostructural particles due to their sizes and peculiarities of their atomic structure reveal such unique physico-mechanical properties that the range of their perspective applications spreads over many human activities, starting from microelectronics to medicine.

In recent years especially increased interest in such technologies of materials production on carbon basis as ones oriented on production of doped carbon nanoparticle modifications (nanotubes, nanoclusters, nanothreads). This gives opportunity to scientists and engineers in noted materials the possibility of aimed control of their natural properties [1].

As the matter of fact, the nanoobject control on nanometric level using nanoparticles with aim to arrange them in rows, signatures and grids is the clue to the production of new functional materials. Correspondingly, for obtaining of building units with different nanometric sizes in the last years many methods of self-assembling and synthesis were developed. In this direction the possibility to control perfectly the self-assembling and synthesis processes of nanoparticles is a serious challenge from the point of view both fundamental and applied investigations.

On the basis of fundamental principles, the process of self-assembling requires the existence of interaction between atoms and clusters, such as thermodynamic and kinetic moving forces, so that it could be realized the organization of atoms and clusters for creation of nanosize domen structures. From this point of view, magnetic nanoparticles deserve a particular interest due to their unique physico-chemical properties and applicability in the new functional materials technologies.

Carbon shells provide both the protection of ferromagnetic impurities from aggressive environment and new unique properties to the hydride nanostructures. The self-assembling of magnetic clusters coated by carbon shells presents just such example which could be used in the contemporary materials, for example, in strong magnets, analytic instruments (nuclear magnetic resonance tomographs) and nanosensors.

The more so, currently magnetic carbon nanoparticles due to their low toxicity are under testing for therapeutic and diagnostic applications.

Last years the magnetic field was applied also for creation of nanoscale materials what resulted in significant achievements in the problem of formation of macro- and microstructure synthesized materials possessing unique properties.

In contrast with other existing self-assembling technologies, the induced by magnetic field ordering defines the formation of magnetic nanoparticles in ordered structures with unique properties. Therefore, the areas of application of carbon magnetic nanoparticles are very large. Sufficient to name such of them as magnetic fluids, plastic glasses stable against scretches, information storage magnetic media, sensors, biomedicine, etc. It should be noted here that in spite of their large perspective for multifunctional applications, the carbon nanoparticles doped by ferromagnetic clusters have not studied sufficiently well [2]. 
In this work following aims are addressed:

1) On the bases of obtained nanopowders the preparation of polymer composite films and study of nanoparticle diffusion and self-healing processes in them under combined influence of heating, pressure, steady and alternating magnetic fields.

2) The study self-organization processes in magnetic polymer composite under the influence of a outer steady magnetic field and heating.

\section{Experimental Results and Their Discussions}

To achieve these aims experiments were carried out to study self-healing and self-organization processes in magnetic polymer composites filled with magnetic carbon nanopowders synthesized by CVD method [3]. These processes were facilitated by heating films above the glass transition temperature. Particles in conventional composites are essentially immobile in contrast to polymer nanocomposites (PNC) particularly above the glass transition temperature $T_{g}$. The nanoparticles mobility can affect polymer dynamics resulting in changing of viscosity, modulus, kinetics of the particle-cluster formation, etc. [4].

Tensile measurements have shown that below $T_{g}$ conventional composites and PNCs behave similarly with respect to mechanical properties. But above $T_{g}$ the toughness of PNC can increase by the order of magnitude with increase of temperature. It has been suggested that the mechanism to the toughness enhancement is the mobility of the nanoparticles. The development of self-healing materials and coatings where nanoparticles migrate toward various defect sites, requires a better understanding of the nanoparticle diffusion processes.

The heating of PNC above $T_{g}$ increases mobility of polymer chains what should facilitate boundary diffusion between polymer interfaces and this effect could be visualized using magnetic nanoparticles introduced in polymer. This process could be improved applying additional stimuli, in particular a low-frequency (ac) magnetic field, stationary magnetic field, pressure, heating, separately or in combinations. Such impact stimulate self-organization processes between prepared films in result of which one could produce "glued" to each other films without using other type glues and polymer melting temperature. As research object for one of the aims of this work was the development of simple technology of production of carbon nanoparticles doped by ferromagnetic clusters and study of their morphology and composition.

Generally the most known method of production of ultra-small cluster-size particles is based on the condensation principle from gas or vapor states, with the possibility of obtaining the product on a substrate or as a powder. In the same time, for the vapor production different methods were used: laser ablation, thermal, electron-beam and ion irradiation.

In particular, for the production of nanopowders and nano-coatings on carbon basis it is used the chemical vapor deposition (CVD) method-mainly along with application of hydrogen reduction process of volatile chlorides. Traditional 
methods of production the pyrolytic carbon by the way of thermal decomposition of carbon containing compounds on the heated surfaces are also well known but all of them have some significant defects: at formation of coatings-low growth rate, high temperature of process $\left(\geq 1300^{\circ} \mathrm{C}\right)$, insufficient quality of obtained coating structures, impossibility to carry out the doping process in one cycle. The analysis of tendencies of development of the contemporary natural science and technology the experimental and theoretical investigations of carbon nanoparticles, nanotubes, nanothreads and doped by them new polymer composites should gain priority. The carbon nanoparticles doped by magnetic ( $\mathrm{Co}, \mathrm{Fe})$ nanoclasters with mean sizes in the range $50-100 \mathrm{~nm}$ were synthesized by technology using the combination of pyrolysis of ethylene spirit (and other hydrocarbons) vapor pyrolysis and CVD process in the mode of recirculation closed cycle with monitored technology parameters [3].

The developed technological process was realized in the installation where in the construction of its reactor and in necessary units for functioning the possibility was foreseen of monitoring such parameters as: vapor content and concentration in reactor zones, catalytic capacity of substrates, partial oxygen pressure. This allows one to carry out technology investigations with aim to establish the optimal technological parameters for production of finely dispersed carbon nanopowders doped with magnetic nanoclusters and, as result, to produce these materials in necessary amounts for investigations and perspective applications.

For the preparation of polymer films it was chosen polyvinylbutyral (PVB) polymer with low $T_{\mathrm{g}} \sim 45^{\circ} \mathrm{C}-55^{\circ} \mathrm{C}$. PVB is a resin mostly used for applications that require strong binding, optical clarity, adhesion to many surfaces, toughness and flexibility.

As filler the produced by us carbon nanopowder doped with magnetic Co nanoclusters (C/Co) was used. For a comparative study we used also the commercial Co nanopowder (Sun firm, USA $28 \mathrm{~nm}$-mean diameter). The Co doped carbon nanopowders were mainly used at their concentrations in polymeric composite in range $10-50 \mathrm{wt} \%$, but for Co nanopowder filled polymer composite concentration was $20 \mathrm{wt} \%$.

Firstly the $10 \%$ alcohol solution was prepared, then this solution was poured into teflon press-forms (Figure 1) and after their drying during 48 hours the 0.3 - $0.5 \mathrm{~mm}$ thickness films were obtained. The filled composites were prepared in the following way: magnetic nanopowders were taken in corresponding proportion (in terms of dry weight) and the usual mixing of PVB with alcohol took place followed by the ultrasound $10-15$ min treatment for the destruction of magnetic nanopowder agglomerates. Next, after thorough mixing during 7 - 10 min magnetic polymer composite films were obtained similar unfilled ones in teflon press-forms. Then sample films were cut from these films (Figure 2).

The trial films were places on each other and subjected to following actions:

1) Impact of pressure and temperature in a vacuum cabinet at pressure $p=0.5$ $\mathrm{MPa}$ and temperature $T=80^{\circ} \mathrm{C}-85^{\circ} \mathrm{C}$ during time $t=2.5$ hours. 


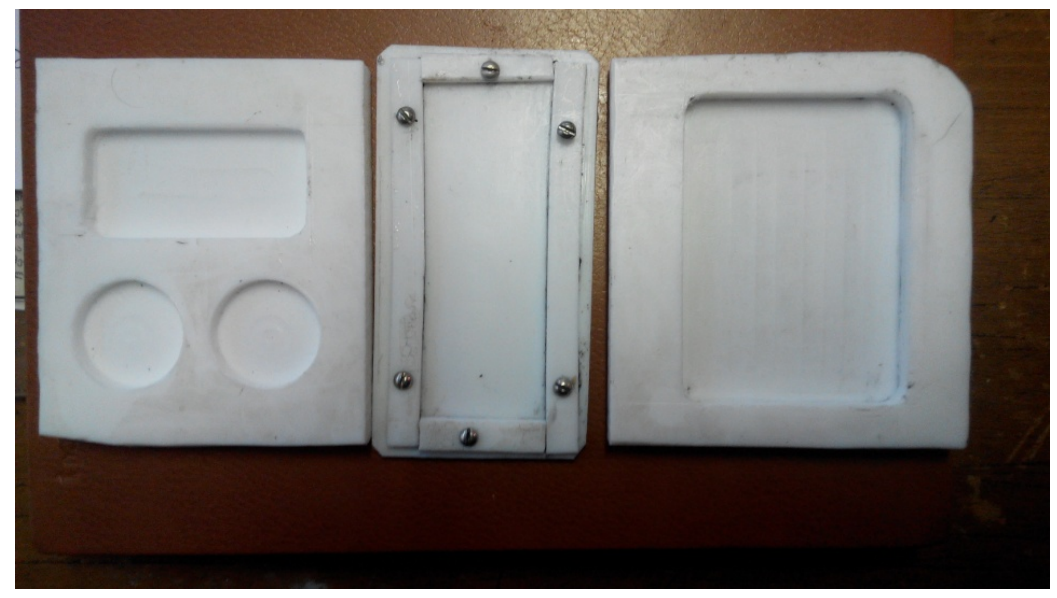

Figure 1. Teflon press-forms for preparation different size films.

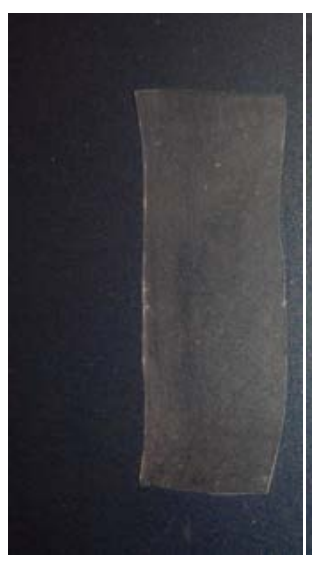

(a)

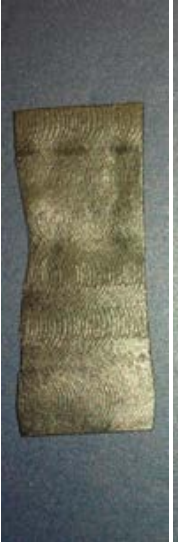

(b)

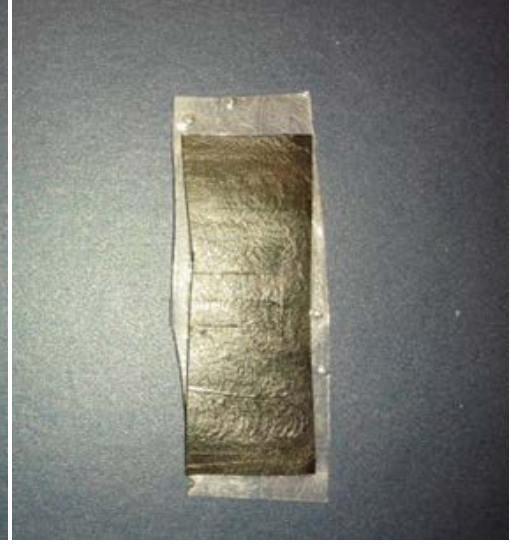

(c)

Figure 2. Filled (a) and unfilled, (b) films separately and jointly, (c) with filled film above unfilled one.

2) Impact of pressure and temperature and stationary magnetic field in a vacuum cabinet at $p=0.5 \mathrm{MPa}$ and temperature $T=80^{\circ} \mathrm{C}-85^{\circ} \mathrm{C}$. During the experiment pressure included a contribution from the magnet weight. The duration of experiment was $t=2.5$ hours.

3) In above $a$ and $b$ items, the obtained double films were subjected to the excitation by a low-frequency magnetic field during $t=2$ hours.

In Figure 3, the vacuum cabinet is presented in turn on position, with placed inside samples during the pressure and temperature action.

During an experiment temperature reaches $85^{\circ} \mathrm{C}$, pressure was approximately $0.5 \mathrm{MPa}$. In the teflon form first of all magnets were placed, then an unfilled film and, finally, polymer composite film on which pressure acts. Then the low-frequency generator turns on with cooling of coil by water and heating following which the combined actions is realized which could continue several hours till the double layer sample is glued by diffusion of nanoparticles.

In Figure 4 the experimental setup is mounted accordingly the experimental scheme is presented. 


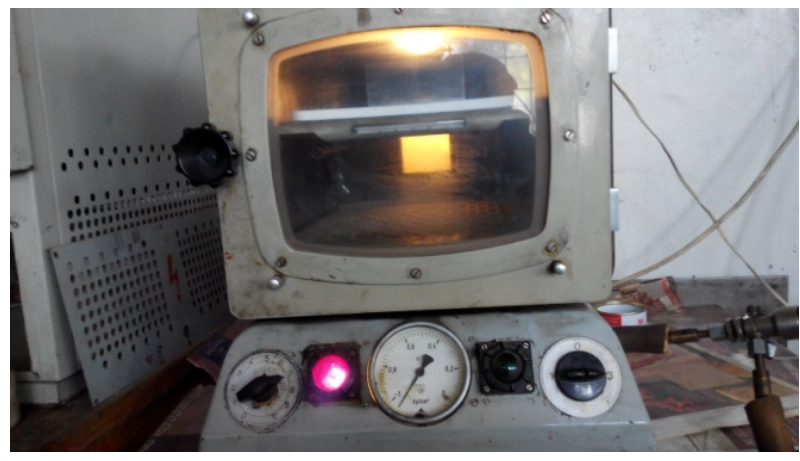

Figure 3. The vacuum cabinet with samples under the testing.

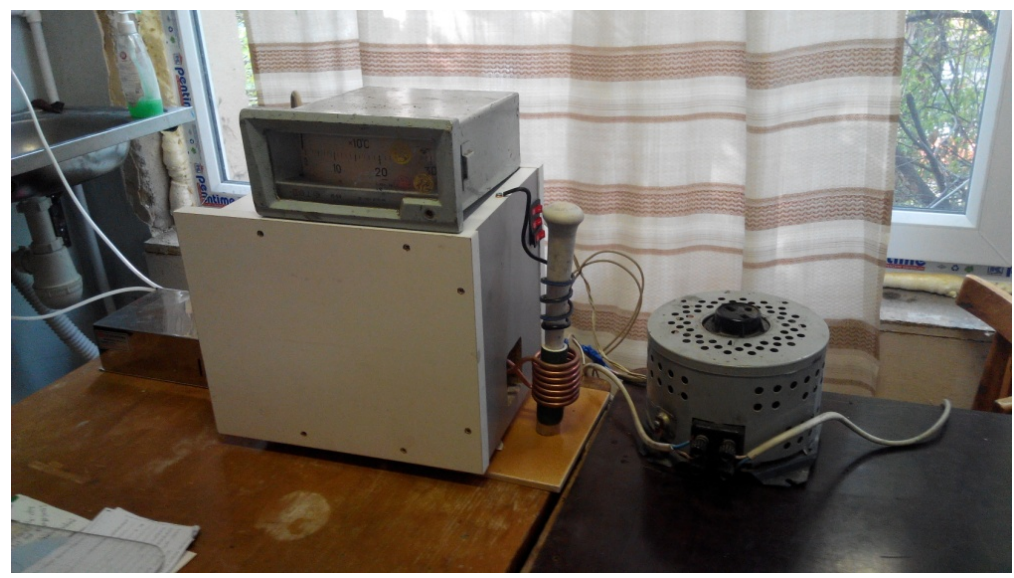

Figure 4. The experimental setup.

In Figure 5, neodymium magnets used in experiments are seen, as well as in Figure 6 the press form for fabrication of samples is shown.

To study the self-organization of polymer nanocomposites it is necessary to increase our understanding of nanoparticles diffusion processing taking place in them [1].

As it was noted, nanoparticles in usual composites, in difference with polymer nanocomposites (PNC) are essentially immoble is particular as compared with diffusion processes rate in PNC above the temperature of glass transition $T_{\mathrm{g}}$.

Besides it, the knowledge of nanoparticles diffusion processes is important for the development of self-healing materials and coatings where nanoparticles migrate to different defect sites.

For the study of cobalt doped carbon nanopowder diffusion processes a series of above described polymer samples were prepared. A sample is a $0.3 \mathrm{~mm}$ thickness polymer nanocomposite film (polymer filled with $\mathrm{C} / \mathrm{Co}$ nanoclasters, the nanoparticle composition was in range of $10-50 \mathrm{wt} \%$ ) and $0.5 \mathrm{~mm}$ thick pure polymer substrate with total pile dimensions $28 \times 10 \times 0.8$ (Figure 7).

The diffusion processes of C/Co nanoparticles across interface between layers at different stimuli at $T>T_{\mathrm{g}}$ : stationary magnetic field, alternating magnetic field $(20 \mathrm{kHz}, 1 \mathrm{~kW}$ power), and their combinations, the adhesion and, consequently, related with the adhesion self-healing processes were studied. 


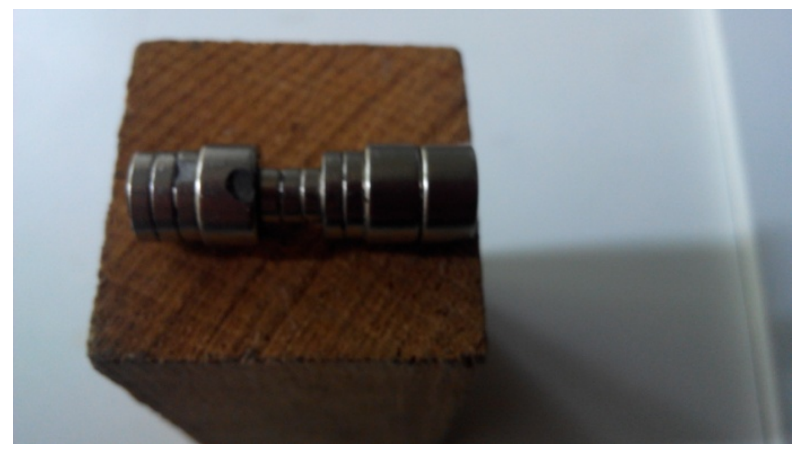

Figure 5. Neodymium magnets used in experiments.

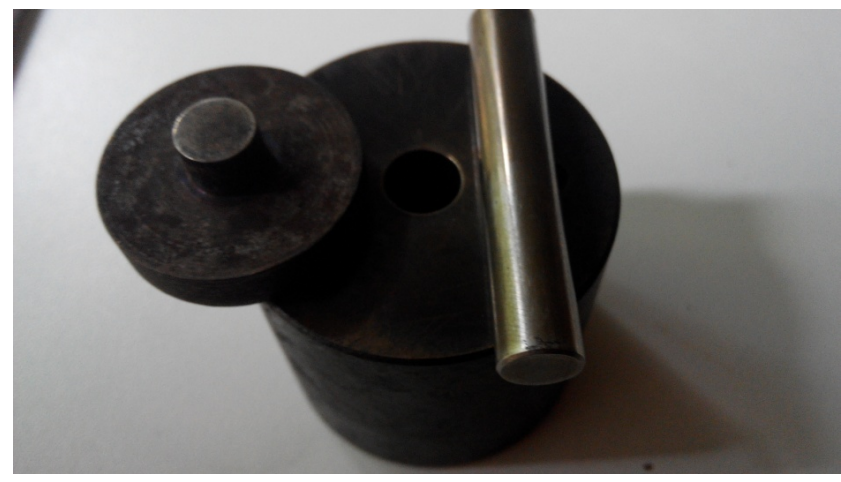

Figure 6. Press form for samples fabrication.

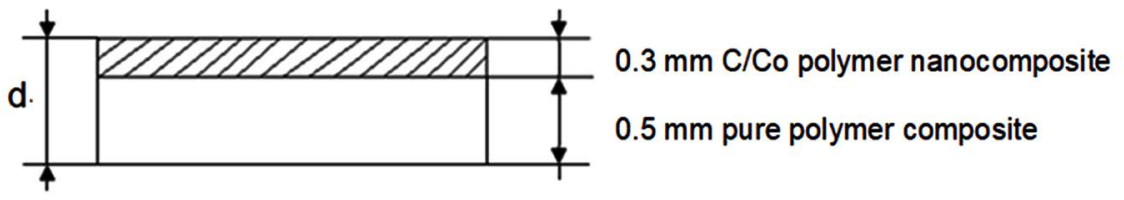

Figure 7. Experimental polymer pile.

To study these processes the layer by layer removal was carried out starting from pure polymer layer down to composite one using a fine grinding. This grinding process was followed by the simultaneous control of sample resistance.

The method appeared to be very visual and effective and allowed one the assessment of nanoparticle mobilities at different excitations and their combinations. It was revealed that the best diffusion of nanoparticles and adhesion of composite film was obtained at the combined effect of temperature, steady and alternating magnetic fields.

In Figures 8-13, the distribution of resistance along a sample is presented at fine grinding describing the degree of penetration of nanoparticles in the polymer substrate.

The good conductivity of polymer composite was stipulated by the presence in the composite of the cobalt carbon nanoparticles at studied C/Co wt. 50\% polymer nanocomposite/pure polymer pile.

During treatment of sample shown in Figure 11, the peeling of upper composite layer was observed what was not observed at other cases. 


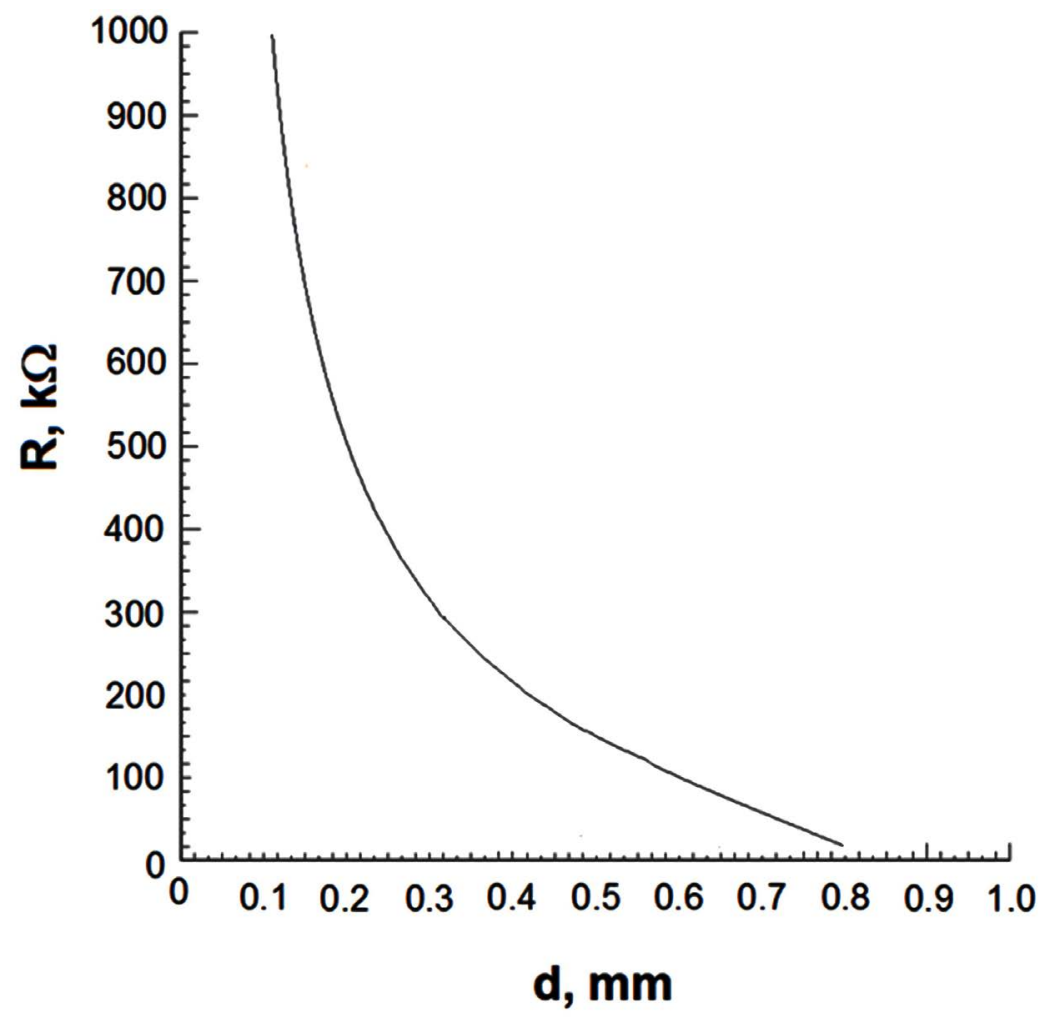

Figure 8. The resistance dependence on the sample thickness. The treatment took place at application of steady and alternating magnetic fields at $T=85^{\circ} \mathrm{C}$.

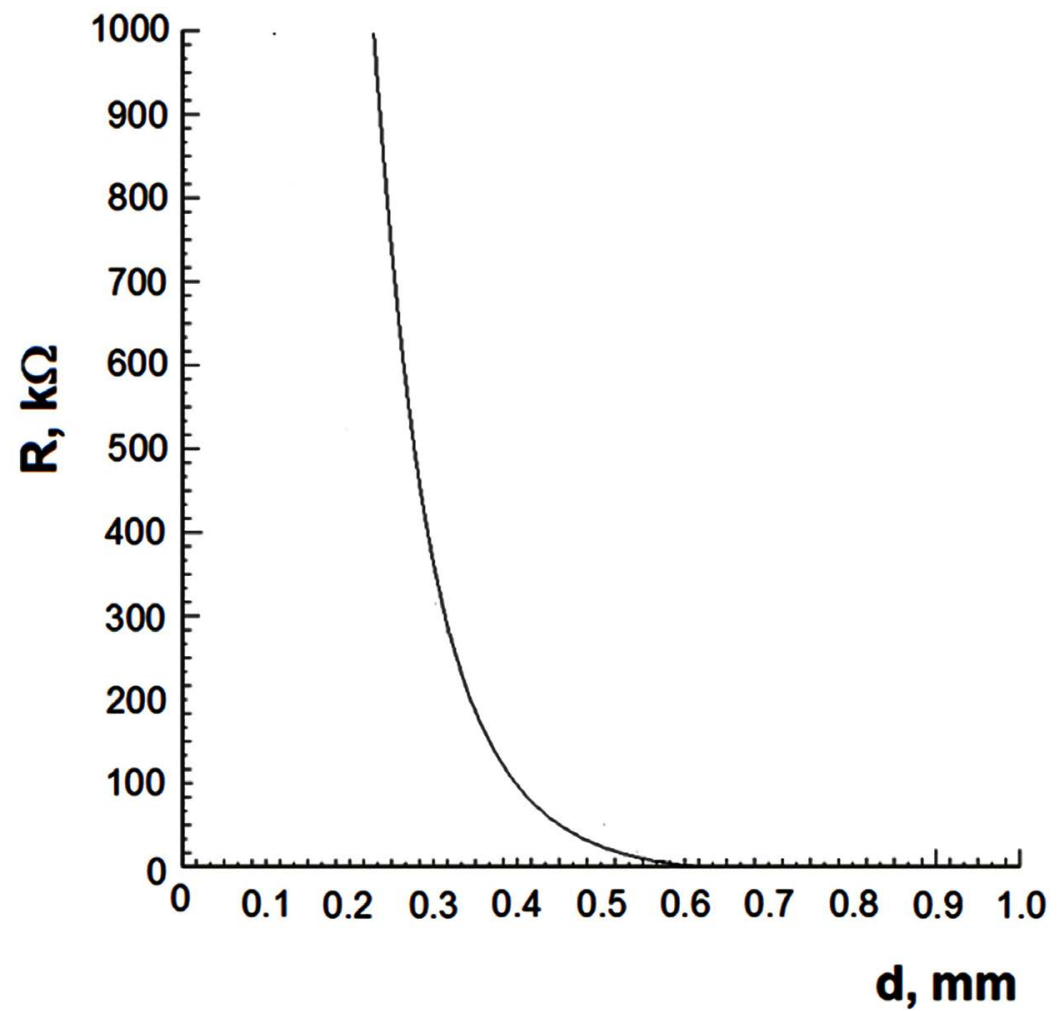

Figure 9. The resistance dependence on the sample thickness. The treatment was made by steady magnetic field at $85^{\circ} \mathrm{C}$. 


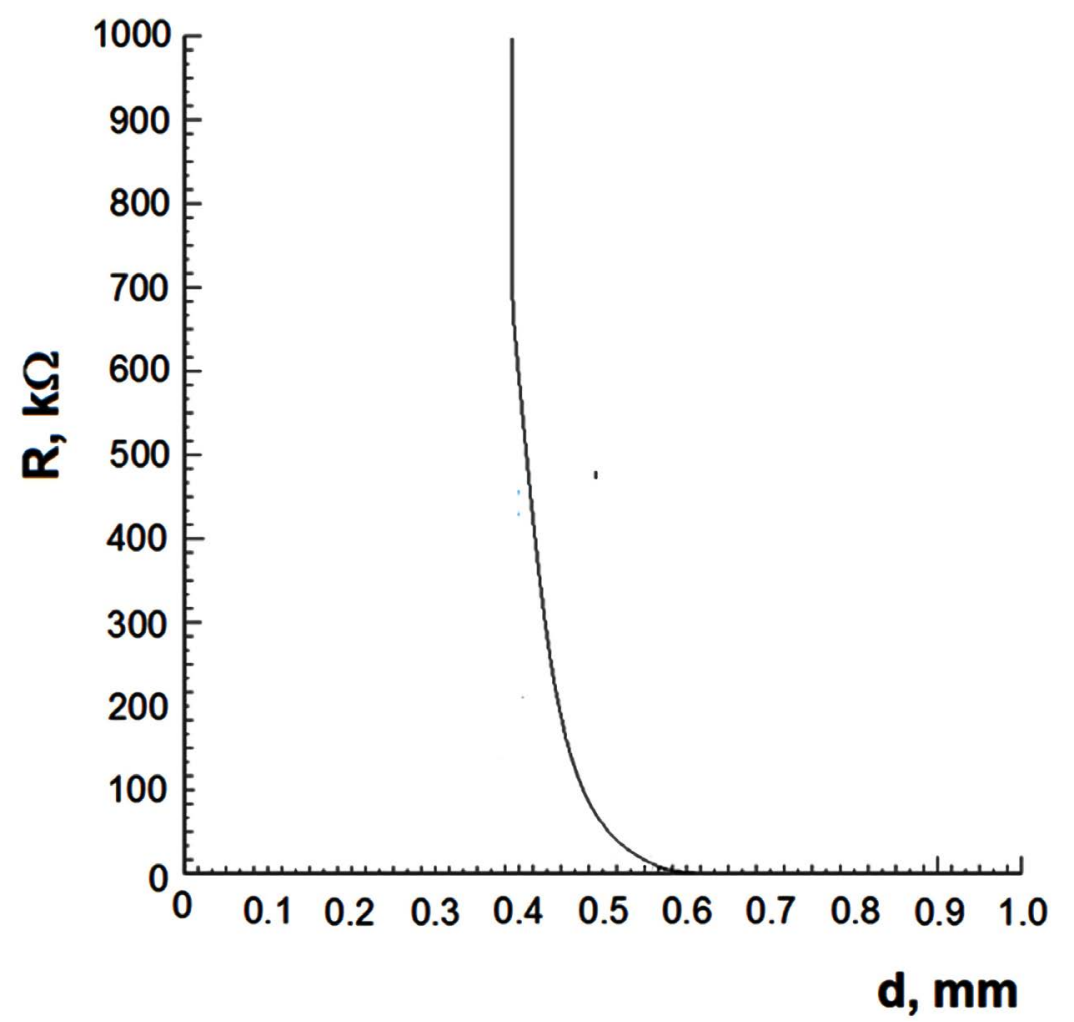

Figure 10. The resistance dependence on sample thickness. The treatment is without magnetic field at $85^{\circ} \mathrm{C}$.

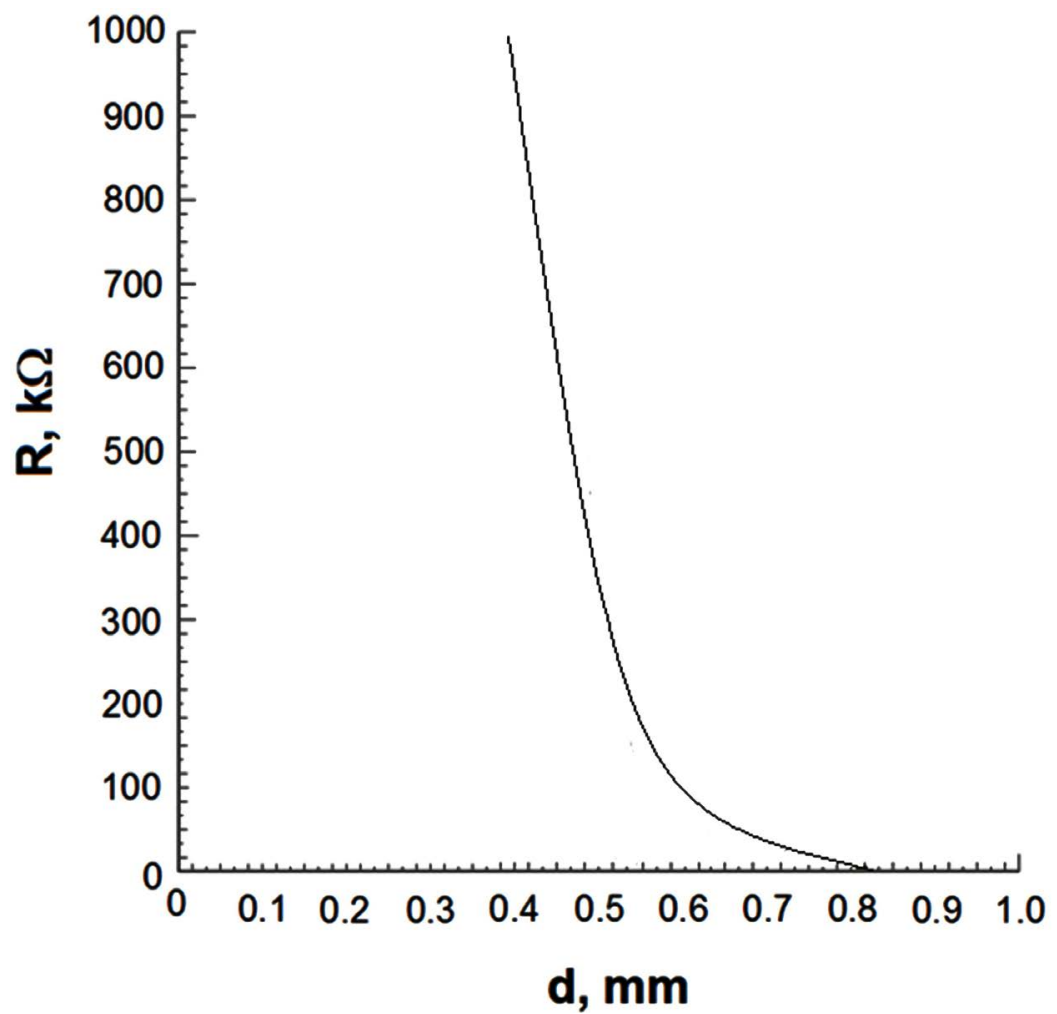

Figure 11. The resistance dependence on sample thickness. Treatment is by alternating magnetic fields at $T=85^{\circ} \mathrm{C}$. 


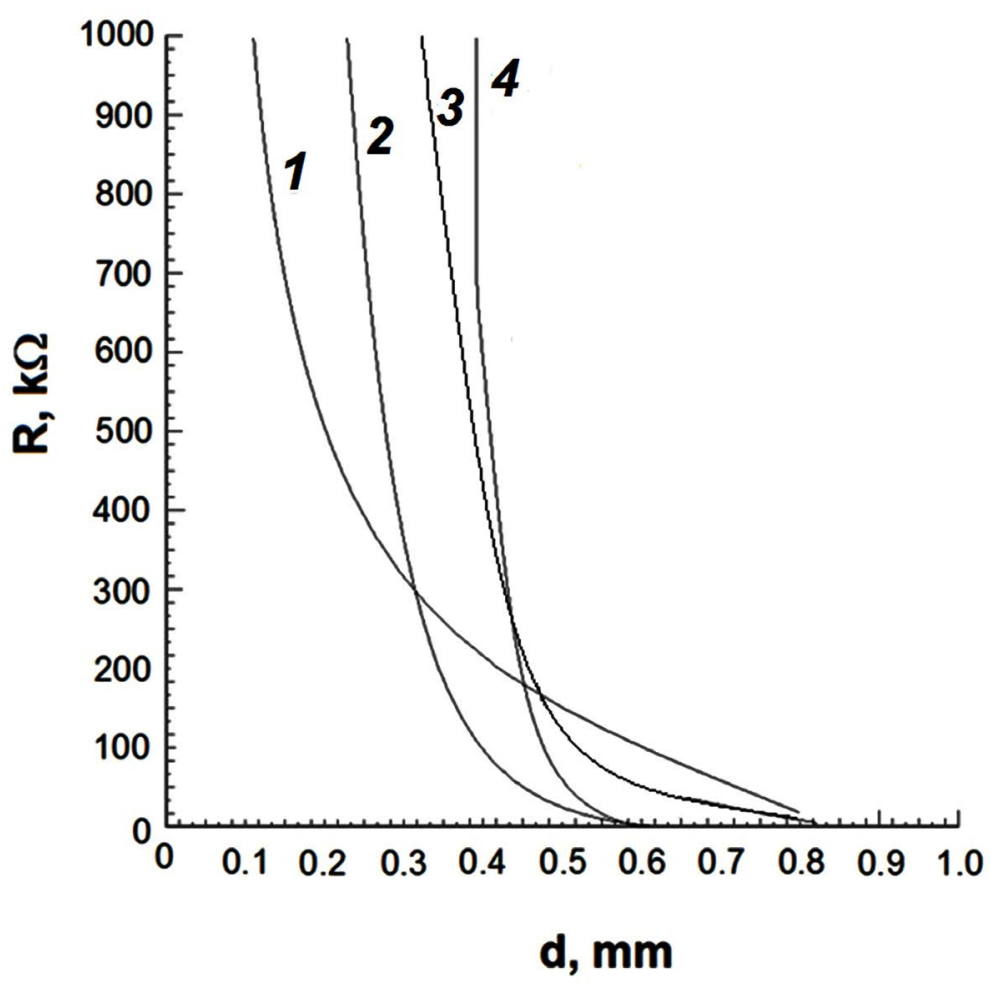

Figure 12. The resistance dependence on sample thickness (summary): Curve 1 corresponds to one given in Figure 8; Curve 2 corresponds to one given in Figure 9; Curve 3 corresponds to one given in Figure 10; Curve 4 corresponds to one given in Figure 11.

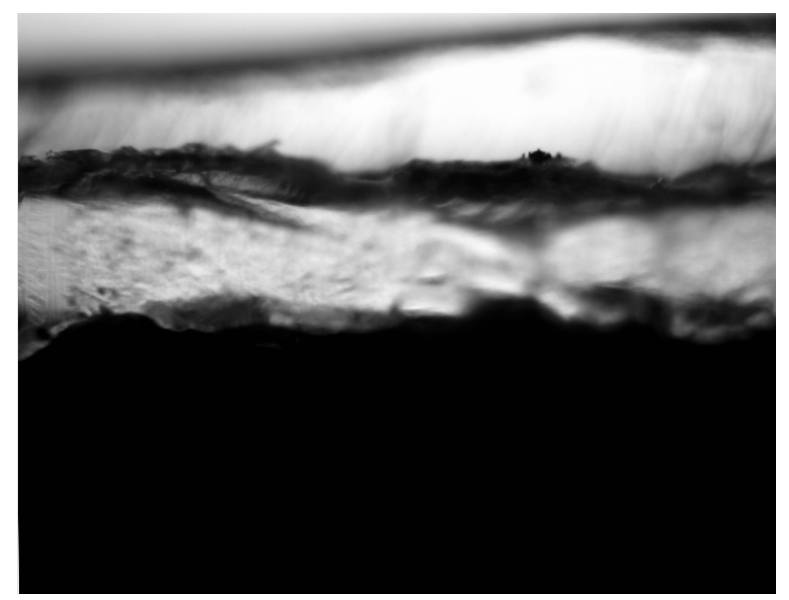

Figure 13. Treatment of C/Co wt. $50 \%$ sample at steady magnetic field and at $T=85^{\circ} \mathrm{C}$ during 2.5 hours.

Note also that in a similar experiment when as a filler was used commercial Co nanoparticles ( $22 \mathrm{~nm}$ is mean diameter) passivated by oleic acid the composite sample proved to be non-conductive due to non-conductive polymer coating of Co nanoparticles and absence of conductivity of pure polymer.

For the control of nanoparticle diffusion in both polymer composites (with C/Co and Co nanoparticless) we used optical microscope OMAX with up to 2500 enlargement. 
For these optical measurements a small plate was cut from a sample with the width $1 \mathrm{~mm}$ and taken pictures of its transverse cutting during any type of influence or their combinations.

For different cases pictures are given in Figures 13-15.

The results obtained by this method are in agreement with resistance measurements ones.

In result of carried out experiments the conclusion was made that the stimulated diffusion of nanoparticles is most fast when temperature and both magnetic field (steady and alternating) acted in combination.

To study further self-assembly processes in this polymer composites at different concentrations of carbon magnetic nanopowder we used a simple method from work [5]. In this case circular shape polymer composite samples (diameter 20 - $32 \mathrm{~mm}$, thickness $1 \mathrm{~mm}$ ) were exposed to a magnetic field which was provided by two attached permanent neodymium magnets and heating (Figure 16), and temperature $85^{\circ} \mathrm{C}$ during two hours.

Resulting self-assembly of C/Co nanopowders caused changing of their concentration and modulation of local resistance $R$ along the radius of sample which was measured by two-contact method as in work [5] (Figures 17-22).

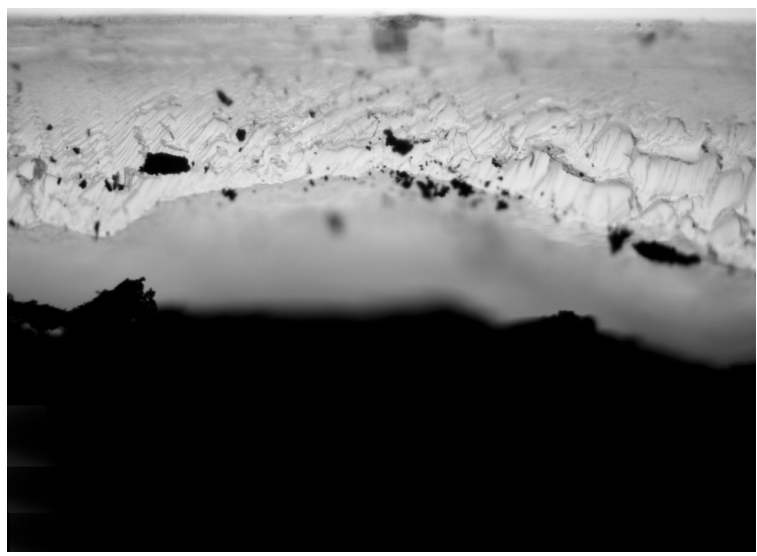

Figure 14. Treatment of C/Co wt.50\% sample without magnet at $T=85^{\circ} \mathrm{C}$ during 2.5 hours.

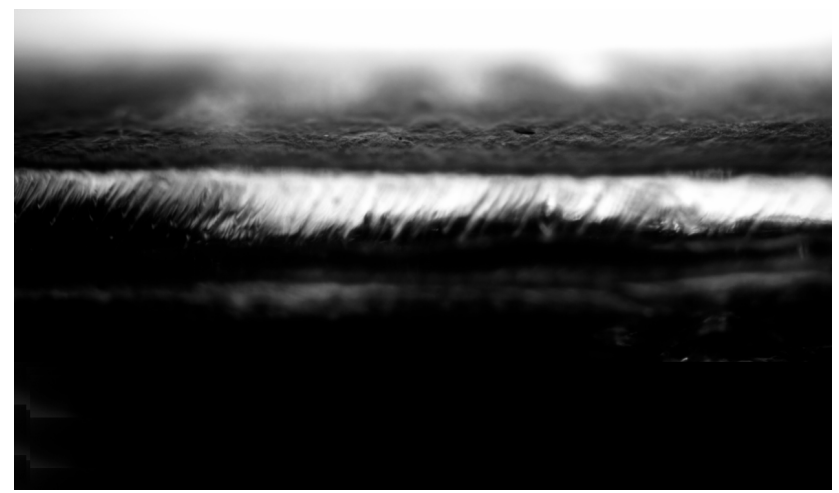

Figure 15. Treatment of Co/C wt. 50\% sample by both type magnetic fields at temperature $T=85^{\circ} \mathrm{C}$ during 2.5 hours. 


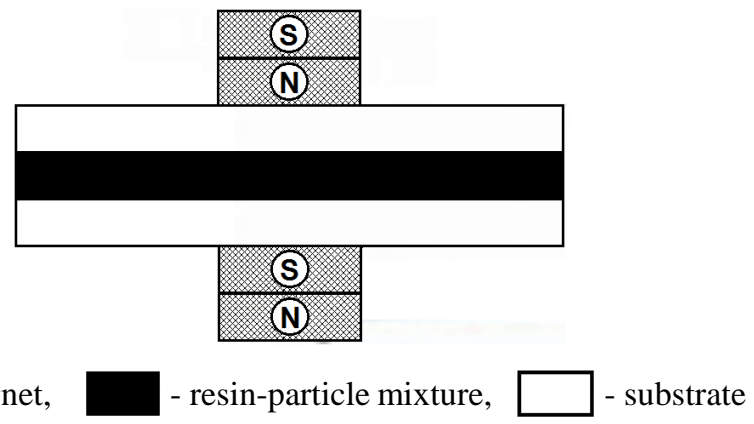

- Magnet,

Figure 16. Geometry of samples.

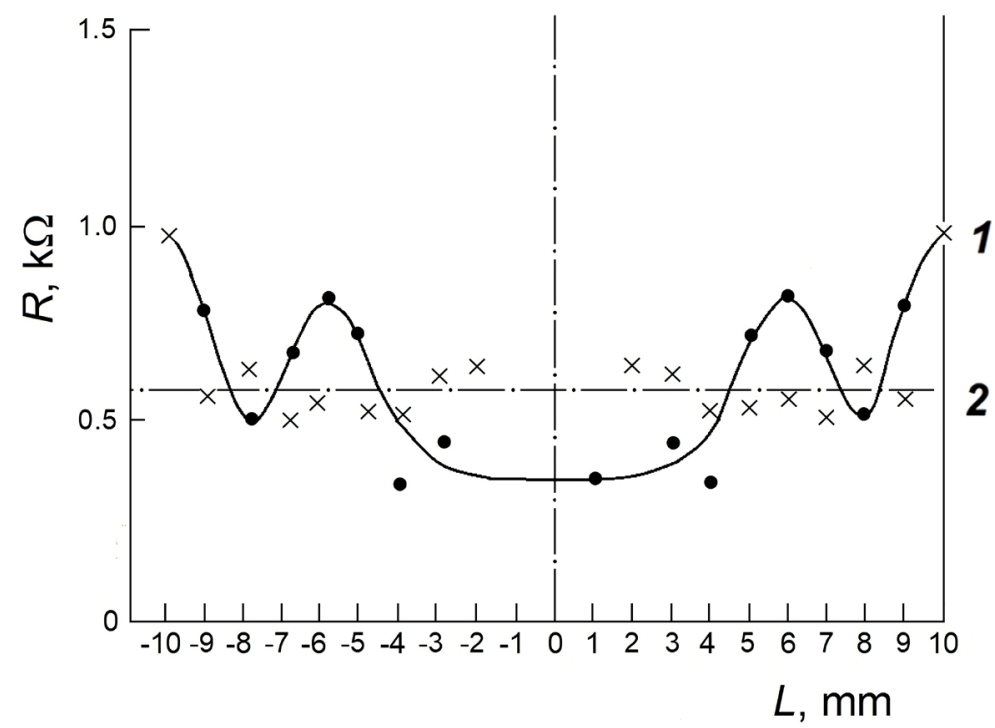

Figure 17. Polymer composite C/Co, wt. $30 \%$; 1: resistant distribution in the sample as result of treatment; 2 : resistant in the sample before the treatment.

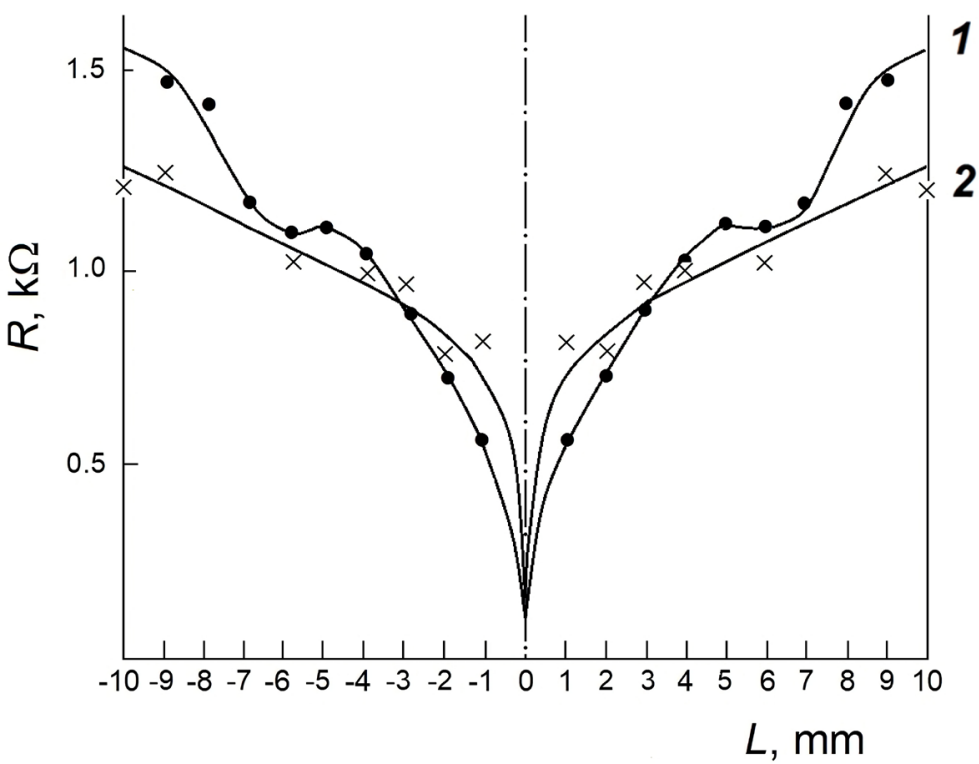

Figure 18. Polymer composite C/Co, wt. 30\%; 1, 2: treated and untreated samples, correspondingly. 


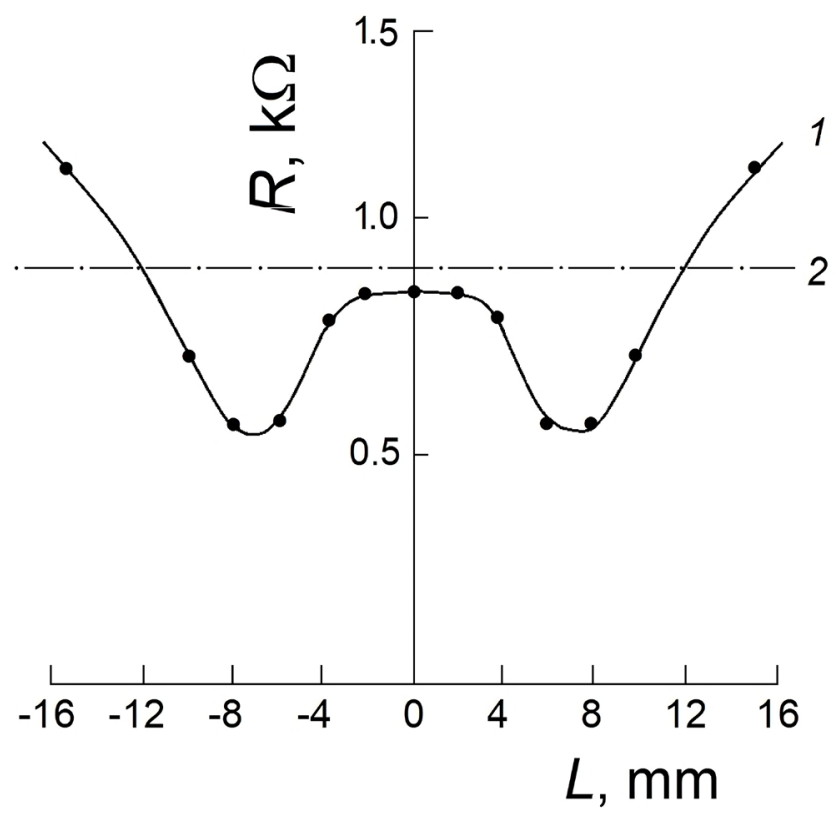

Figure 19. Polymer composite C/Co, wt. 20\%; 1, 2: treated and untreated samples.

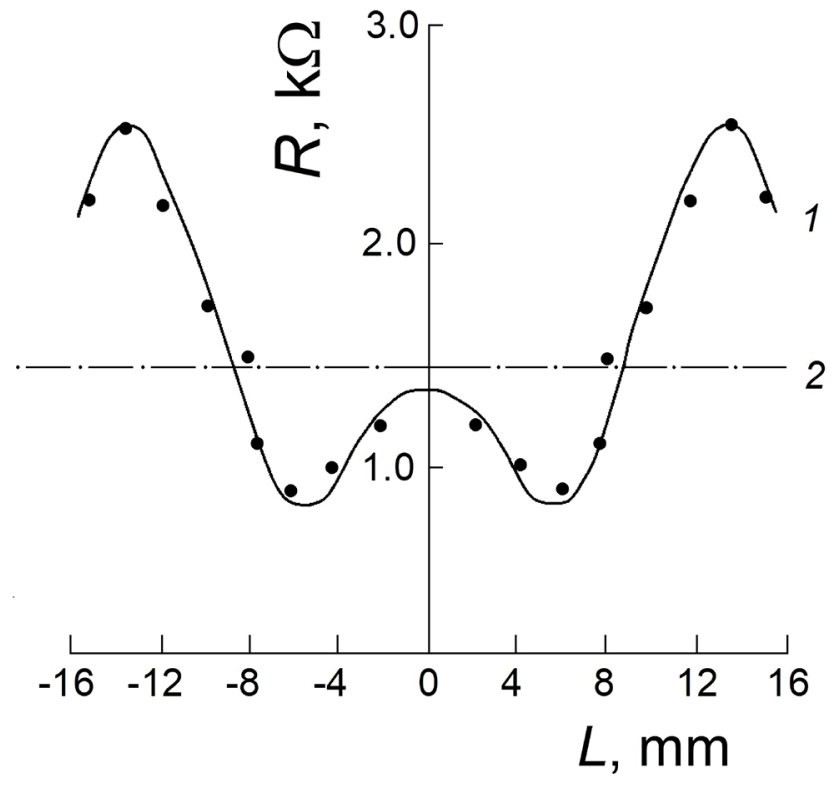

Figure 20. Polymer composite C/Co, wt. 15\%; 1, 2: treated and untreated samples.

Resistance was measured between points separated by $2 \mathrm{~mm}$ along radius in all following cases besides Figure 18 where resistance was measured between sample's center and points on radius with the interval $2 \mathrm{~mm}$.

Based on the obtained experimental results, we were able to investigate the self-assembling processes in the magnetic polymer nanocomposite films synthesized by the developed technology using the carbon magnetic nanopowders fabricated by the method of [4] under the combined influence of magnetic field and heating using sensitive and simple non-contact RF resonant magnetometry method [5]. 


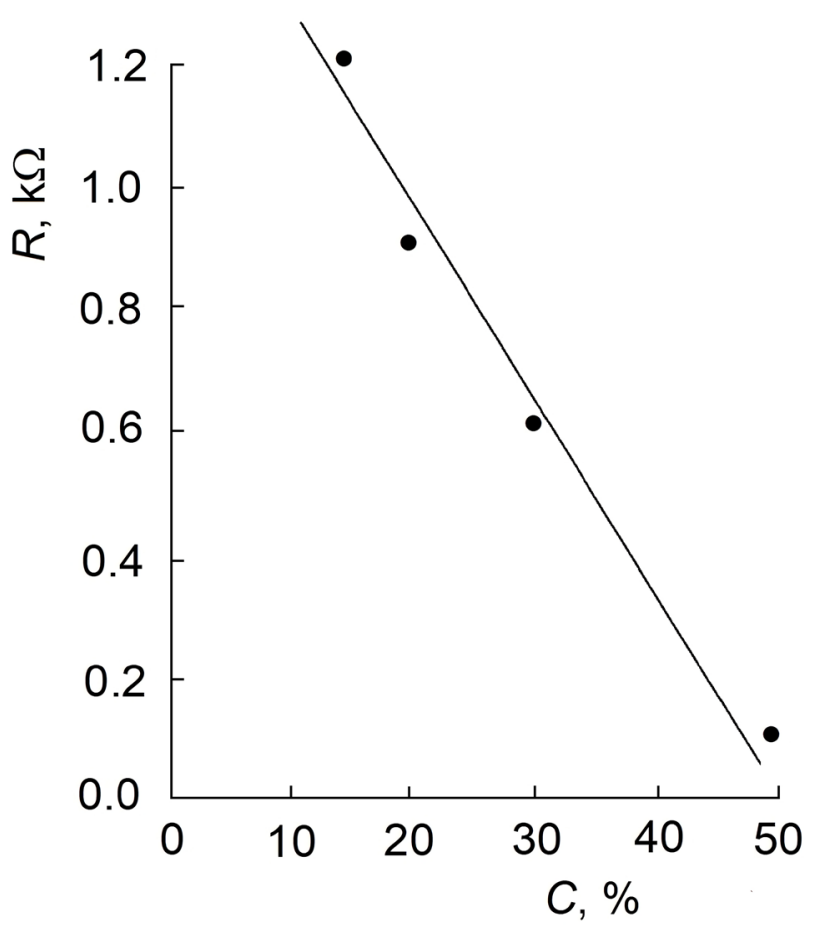

Figure 21. The dependence of resistance of initial untreated samples on the nanopowder concentration $\mathrm{C}$.

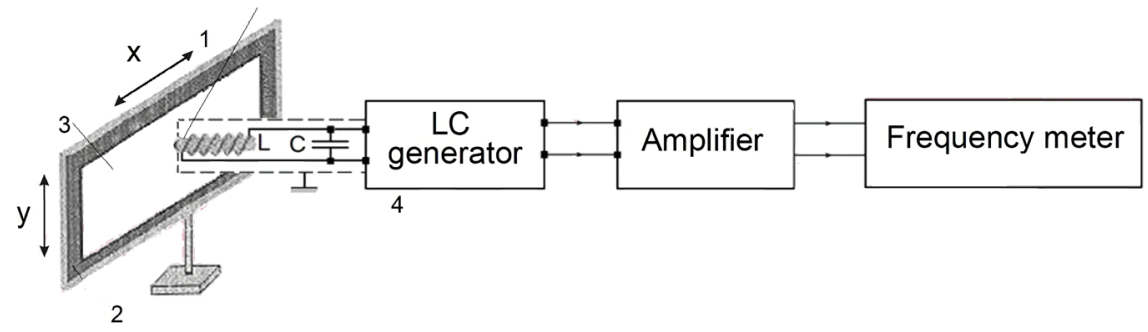

Figure 22. The setup for study of the self-assembling processes in the polymer magnetic nanocoposite films. 1: ferrite probe; 2 : frame; 3: polymeric magnetic nanocomposite film; 4: LC generator.

In the inductive coil of the RF resonant contour of LC-generator a cylindrical tipped ferrite rod is used as a probe. The investigated disk shape magnetic polymer nanocomposite film is displaced relatively the immovable ferrite probe tip. The scanning of the film surface is realized along the previously marked disk diameter.

During the scanning of magnetic polymer composite film by moving ferrite probe the variation of $\mathrm{RF}$ resonant magnetometer frequency is observed due to the change of the cobalt nanocluster concentration in the polymer composite film and related with it change of the coil inductance.

For example, we present the results of self-assembly processes measurements of carbon cobalt nanoclusters in polymer nanocomposite film under the influence of the magnetic field of neodymium magnet with diameter $D=12 \mathrm{~mm}$ and heating (Figure 23 and Figure 24), which are similar to the results obtained during 


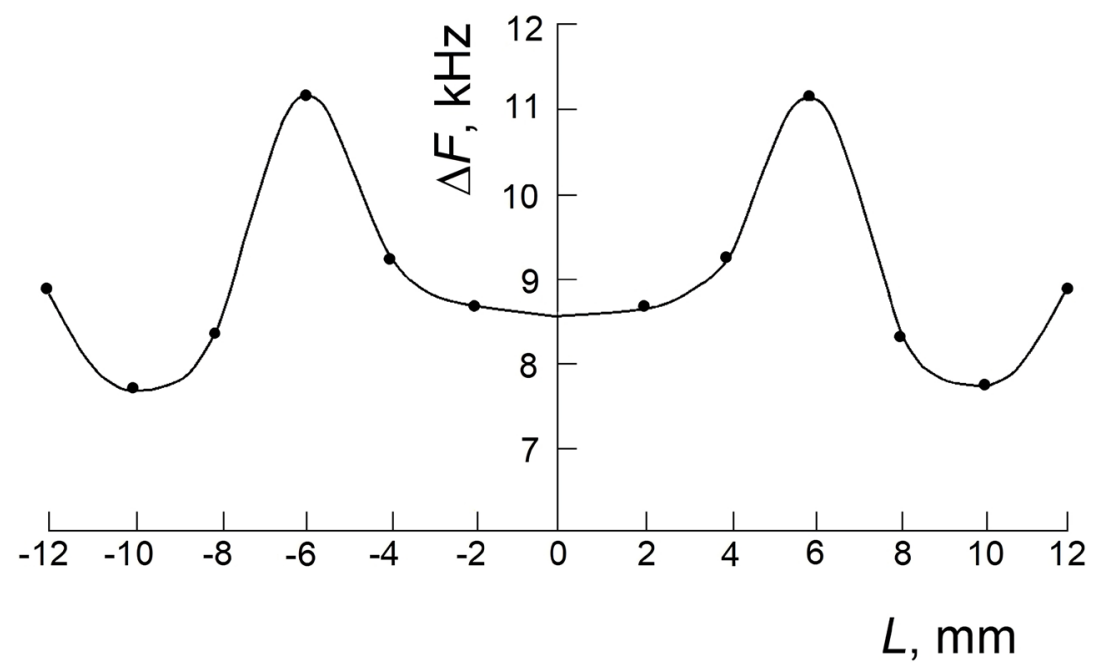

Figure 23. Dependence of the RF resonant magnetometer oscillation frequency at displacement of ferrite probe along the diameter of $\mathrm{C} / \mathrm{Co}$, wt. $50 \%$ carbon cobalt polymer nanocomposite disk film, $\mathrm{D}=24 \mathrm{~mm}$.

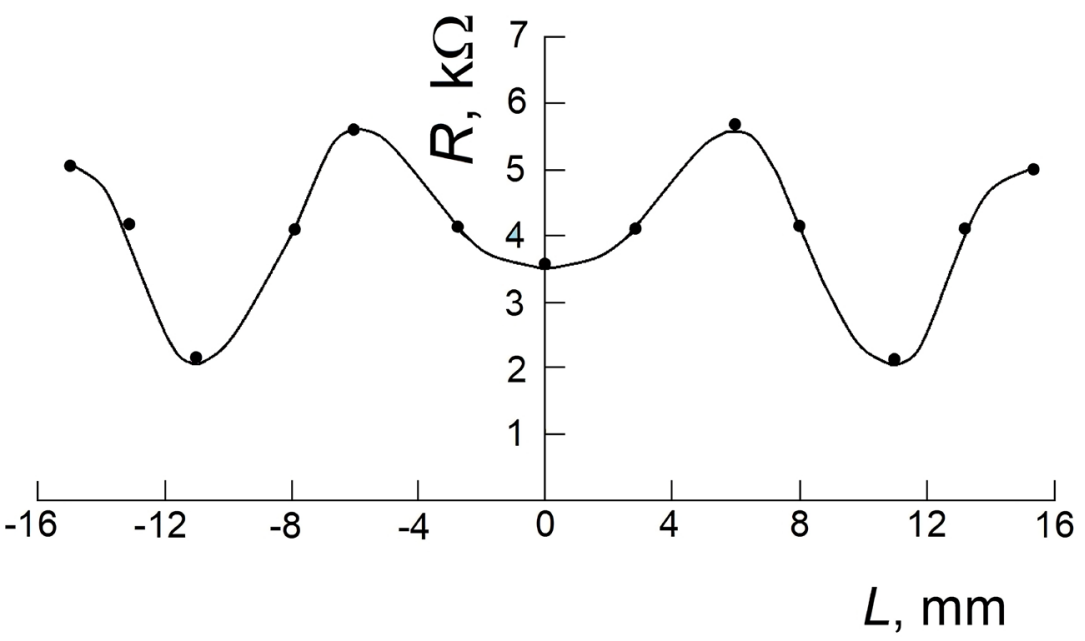

Figure 24. Dependence of the RF resonant magnetometer oscillation frequency at displacement of ferrite probe along the diameter of C/Co, wt. $20 \%$ carbon cobalt polymer nanocomposite disk film, $\mathrm{D}=30 \mathrm{~mm}$.

the study of self-assembly processes by the two-contact electrical resistance measurements of the same samples.

In work [6] the possibility to repair physically separated phermoplastic polymethacrylates containing superparamagnetic nanoparticles using oscillating magnetic field (OMF) was shown without any chemical intervention while maintaining film mechanical properties and the repair can be repeated several times on the same area.

The first step in this development was to prepare polymer films with uniformly dispersed magnetic nanoparticles. This uniformly dispersed superparamagnetic nanoparticles dispersed in the polymer matrix provides an opportunity for designing self-healing materials with a magnetic signature. It was shown [6] that 
when OMF is applied, the films restore their mechanical properties. This was attributed to the oscillating magnetic $\gamma-\mathrm{Fe}_{2} \mathrm{O}_{3}$ nanopartices, thus resulting in an amorphous flow in the damaged region and repair. It should be also noted that the films retain their dimensional stability. As OMF excites the magnetic moment in magnetic nanoparticles, energy resulting from the Neel and Brownian relaxation is converted to thermal energy.

Accordingly work [7] application of stationary magnetic field on polymer composite containing mixture of carbon black and magnetite nanoparticles resulted in diffusion of oxide particles toward the magnetic field lines and their alignment. The magnetic stimulated iron oxide particles put pressure on the nonmagnetic carbon black particles enhancing particle connection leading to decreased resistivity.

Similar this, the pressure and temperature also contribute to the increased diffusion of magnetic nanoparticles in direction to defect areas. Results of cited works could be used to understand effects observed in this work. As a result of combined stimulated diffusion of C/Co nanoparticles prepared films show good self healing and adhesion properties as well as good electric conductivity due to the passivated conducting carbon coated magnetic Co nanoclasters. These effects were most intensive in the case of combined application of alternating and stationary magnetic fields at temperatures above the temperature of glass transition.

\section{Conclusion}

The self-healing and self-organization processes were studied in the magnetic polymer nanocomposites synthesized on basis of carbon nanoparticles doped by cobalt nanoclasters, synthesized by original CVD technology developed by authors. These processes were taking place under combined stimulated diffusion of magnetic nanoparticles by outer alternative and steady magnetic fields, as well as heating and pressure. Polymeric composite samples have good electric and adhesive properties and are perspective for potential practical applications.

\section{Conflicts of Interest}

The authors declare no conflicts of interest regarding the publication of this paper.

\section{References}

[1] Müller, K., Bugnicourt, E., Latorre, M., Jorda, M., Sanz, Y.E., Lagaron, J.M., Miesbauer, O., Bianchin, A., Hankin, S., Bölz, U. and Pérez, D. (2017) Review on the Processing and Properties of Polymer Nanocomposites and Nanocoatings and Their Applications in the Packaging, Automotive and Solar Energy Fields. Nanomaterials, 7, 74-121. https://doi.org/10.3390/nano7040074

[2] King, V.R. (2007) Nanotechnology Research Advances. Nova Science Publishers, Inc., New York, 187p.

[3] Gegechkori, T., Mamniashvili, G., Kutelia, E., Rukhadze, L., Maisuradze, N., Eristavi, B., Gventsadze, D., Akhalkatsi, A., Gavasheli, T., Daraselia, D., Japaridze, D. and 
Shengelaya, A. (2015) Technology for production of Magnetic Carbon Nanopowders Doped with Iron and Cobalt Nanoclusters. Journal of Magnetism and Magnetic Materials, 373, 200-206. https://doi.org/10.1016/j.jmmm.2014.05.007

[4] Grabowski, C.A. and Mukhopadhyay A. (2014) Size Effect of Nanoparticle Diffusion in a Polymer Melt. Macromolecules, 47, 7238-7242.

https://doi.org/10.1021/ma501670u

[5] Aneli, J., Nadareishvili, L., Mamniashvili, G., Akhalkatsi, A. and Zaikov, G. (2012) Gradiently Anisotropic Conducting and Magnetic Polymer Composites. Chemistry \& Chemical Technology, 6, 285-289. https://doi.org/10.23939/chcht06.03.285

[6] Corten, C.C., and Urban, M.W. (2009) Repairing Polymers Using Oscillating Magnetic Field. Advanced Materials, 21, 5011-5015.

https://doi.org/10.1002/adma.200901940

[7] Mette, C., Fischer, F. and Dilger, K. (2015) Carbon Black Nanoparticle Alignment Using Magnetic Particles Creating Local Percolation Spots for Electrical Conductivity in Structural Adhesives. Proceedings of the Institution of Mechanical Engineers, Part L: Journal of Materials. Design and Applications, 229, 166-172.

https://doi.org/10.1177/1464420713514958 